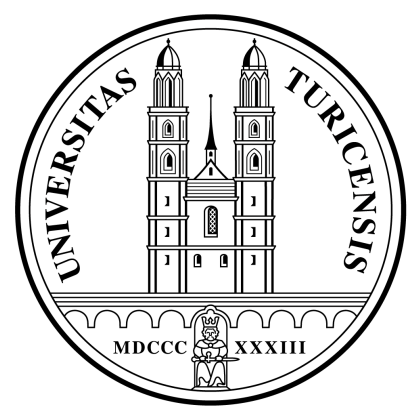

Institute for Empirical Research in Economics

University of Zurich

Working Paper Series

ISSN 1424-0459

Working Paper No. 96

Excess Demand Functions with Incomplete Markets A Global Result

Takeshi Momi

January 2002 


\title{
Excess Demand Functions with Incomplete Markets - A Global Result *
}

\author{
Takeshi Momi ${ }^{\dagger}$ \\ I.E.W. University of Zurich
}

January, 2002

\begin{abstract}
The purpose of this paper is to give a global characterization of excess demand functions in a two period exchange economy with incomplete real asset markets. We show that continuity, homogeneity and Walras' law characterize the aggregate excess demand functions on any compact price set which maintains the dimension of the budget set. JEL classification: D52, C62.
\end{abstract}

\section{Introduction}

Sonnenschein (1973), Mantel (1974), and Debreu (1974) proved that the aggregate excess demand function in the Arrow-Debreu exchange economy is characterized on any compact set of prices by continuity, homogeneity, and Walras' law. This research has been extended to incomplete market models with various asset structures. Similar results have been obtained by Bottazzi and Hens (1996), Gottardi and Hens (1999), Chiappori and Ekeland (1999) and Gottardi and Mas-Colell (2000). However, these results are local; the characterization of the aggregate excess demand is given in a neighborhood of (or at) one price vector. The question naturally arises as to whether these properties

${ }^{*}$ I am grateful to Kazuya Kamiya, Thorsten Hens, an anonymous referee and the associate editor for useful suggestions. Grant-in-Aid for JSPS Fellows is gratefully acknowledged.

${ }^{\dagger}$ Address: I.E.W. University of Zurich, Bluemlisalpstrasse 10, CH-8006 Zurich, Switzerland. Phone: 41-1-634-3798. E-mail: momimomi@e.u-tokyo.ac.jp 
of the aggregate excess demand function in incomplete markets are global in the sense of Sonnenschein-Mantel-Debreu. This question is the subject of the paper. $^{1}$

We focus on an exchange economy with incomplete real asset markets. Our result is therefore an extension of Bottazzi and Hens (1996). In incomplete market economies, demand functions may be discontinuous at critical prices where the dimension of the budget set drops. It is shown that continuity, homogeneity, and Walras' law characterize the aggregate excess demand function on any compact set of prices over which the dimension of the budget set is constant.

While the set of critical prices is clearly negligible, a drawback of our technique is that the number of consumers needed for the characterization is not given by an explicit formula, although it is determined by the number of the commodities, the number of the states and the compact price set on which a candidate excess demand function is defined, and is independent of the particular candidate function. On the other hand, relying on the technique in this paper, the required number of consumers exceed the number of commodities, which is sufficient if the market is complete. Whether this is a general feature of incomplete markets would be an interesting question beyond our scope.

It should be stressed that previous methods used by Bottazzi and Hens (1996) and Debreu (1974) cannot be applied directly to our global problem. Such techniques fail to construct the monotone preference orderings. Overcoming this difficulty is the main technical contribution of the paper. This point is explained in Section 3.

Section 2 gives our main theorem and presents the result on the global characterization of aggregate excess demand as a corollary. Section 3 explains the previously mentioned point, which is the core of the proof of the theorem. The formal proof is given in Section 4.

\section{Main theorem and result}

It is sufficient to state our main theorem rather abruptly; we refer to MasColell (1986) for background. Let $G^{k}\left(R^{n}\right)$ denote the set of $k$ dimensional vector subspaces of $R^{n}$. Here $G^{k}\left(R^{n}\right)$ is a compact smooth manifold called

\footnotetext{
${ }^{1}$ See Hens (2001) for a global characterization in a single commodity model.
} 
the Grassmann manifold. Let $G_{++}\left(R^{n}\right)=\left\{L \in G^{k}\left(R^{n}\right) \mid L \bigcap R_{+}^{n}=0\right\}$. ${ }^{2}$ For $e \in R^{n}$ and $L \in G^{k}\left(R^{n}\right)$, let $\operatorname{proj}_{L}(e)$ denote the projection of $e$ onto $L$. An economy consists of consumers, finite in number, where each consumer is represented by a pair $(\preceq, \omega)$, $\preceq$ a strictly convex, monotone, continuous, complete preference ordering on the consumption set $R_{+}^{n}$ and $\omega$ an endowment vector in $R_{+}^{n}$.

Theorem. Let $G$ be a compact subset of $G_{++}^{k}\left(R^{n}\right)$ such that

$$
(*) \operatorname{proj}_{L}(e)=\operatorname{proj}_{L^{\prime}}(e) \text { for some } e \in R_{++}^{n}, L, L^{\prime} \in G \Rightarrow L=L^{\prime} \text {. }
$$

For any continuous function $L \mapsto z(L)$ defined for $L \in G$ such that $z(L) \in L$, there exists an economy such that for every $L$ in $G, z(L)$ is the aggregate excess demand of some consumers maximizing their utility subject to their net trade lying in $L-R_{+}^{n}$.

In the rest of this section, we demonstrate that this theorem actually solves the problem explained in the introduction. Consider the setup given in Bottazzi and Hens (1996) [p. 52], which is the basic two period exchange economy with incomplete real asset markets $(S$ possible states in period $1 ; l$ goods in each state, so that $R^{n}, n=(S+1) l$, with period 0 as state 0 , is the total commodity space; $J$ real assets $A^{j}, j=1, \ldots, J$, which promise the delivery of a bundle of commodities $A_{s}^{j}=\left(A_{s 1}^{j}, \ldots, A_{s l}^{j}\right)$ if state $s \in\{1, \ldots, S\}$ occurs in period 1 ). The budget set, which the excess demand vectors $z=$ $\left(z_{0}, z_{1}, \ldots, z_{S}\right) \in R^{n}$ have to satisfy, is represented as

$$
L(p)=\left\{\begin{array}{l|l}
z \in R^{n} & \begin{array}{l}
p z=0, \\
\text { for some } \theta \in R^{J} p_{s} z_{s}=\sum_{j} p_{s} A_{s}^{j} \theta^{j}, s=1, \ldots, S
\end{array}
\end{array}\right\},
$$

where $p=\left(p_{0}, p_{1}, \ldots, p_{S}\right) \in R_{++}^{n}$ is a present value price system and $L(p)$ is generically $k=n-(S-J)-1$ dimensional vector subspace in $R^{n}$. Since $L(p)$ satisfies the no-arbitrage condition, $L(p)$ is generically an element of $G_{++}^{k}\left(R^{n}\right)$. As proved by Bottazzi and Hens (1996) [Proposition I and the argument following it, pp. 52-53], if $\operatorname{proj}_{L(p)}(e)=\operatorname{proj}_{L\left(p^{\prime}\right)}(e)$ for $e \in R_{++}^{n}$, then $L(p)=L\left(p^{\prime}\right)$. Therefore the set $\left\{L(p) \mid p \in R_{++}^{n}\right\}$ of budget sets in the incomplete real asset market economy satisfies $(*)$. Now, by identifying a function $p \mapsto z(p)$ with the function $L(p) \mapsto z(p)$ under the assumption of

\footnotetext{
${ }^{2}$ We write the origin of $R^{n}$ as 0 . This should not be confused with $0 \in R$. We also write a single element set $\{x\} \subset R^{n}$ simply as $x$.
} 
homogeneity (i.e., $L(p)=L\left(p^{\prime}\right) \Rightarrow z(p)=z\left(p^{\prime}\right)$ ) of $p \rightarrow z(p)$, the next result is an immediate consequence of our theorem.

Main result. Let $P \subset R_{++}^{n}$ be a compact set such that the dimension of $L(p)$ is $k$ for $p \in P$. For any function $z$ satisfying (i) Walras' law: $z(p) \in L(p)$, (ii) Homogeneity: $z(p)=z\left(p^{\prime}\right)$ if $L(p)=L\left(p^{\prime}\right)$, and (iii) Continuity: $z$ is continuous on $P$, there exists an economy where the aggregate excess demand is $z(p)$ for $p \in P$.

\section{Method of proof}

Both Bottazzi and Hens (1996), who prove the local version of the theorem, and this paper, are generalizations of Debreu (1974), who proves the complete market version $(k=n-1)$ of the theorem. The methods in these three papers may be called the projection-based approach. Debreu (1974) and Bottazzi and Hens (1996) first decompose the candidate function using functions of the form $L \mapsto \beta(L) \operatorname{proj}_{L}(e)$, where $\beta$ is a positive valued continuous function and $e$ is a vector in $R_{+}^{n}$. The individual preference ordering is then constructed so that $\beta(L) \operatorname{proj}_{L}(e)$ is generated as the individual excess demand. The key property of the projection-based construction of the preference ordering is, roughly speaking, that the vector $e-\operatorname{proj}_{L}(e)$ becomes normal to the constructed indifference surface at $\beta(L) \operatorname{proj}_{L}(e)$. In other words, this vector corresponds to the marginal rate of substitution at the point, and hence this vector should be positive to insure monotonicity of the constructed preference ordering.

Let $e$ be a unit vector as in Debreu (1974). For example, let $e$ be the first unit vector and let $n=3$. Consider the case where $k=2$ (complete market). It is easy to see that $e-\operatorname{proj}_{L}(e)$ is positive for any budget set $L$ satisfying the no-arbitrage condition $L \bigcap R_{+}^{3}=0$.

Let us turn to the case of $k=1$ (incomplete market). As an example, let $\bar{L}$ be the line spanned by the vector $(1,-1,1)$. Observe that $\bar{L}$ satisfies the no-arbitrage condition and that $\operatorname{proj}_{\bar{L}}(e)=\left(\frac{1}{3},-\frac{1}{3}, \frac{1}{3}\right)$. Hence, $e-\operatorname{proj}_{\bar{L}}(e)$ is

$\left(\frac{2}{3}, \frac{1}{3},-\frac{1}{3}\right)$, which is not positive. Thus Debreu's method fails in the incomplete market case. It is, however, still possible to make $\bar{e}-\operatorname{proj}_{\bar{L}}(\bar{e})$ positive by taking a suitable $\bar{e}$, and then $\bar{e}-\operatorname{proj}_{L}(\bar{e})$ is positive for $L$ in a neighborhood of $\bar{L}$. This is the way Bottazzi and Hens (1996) obtained their local result applying Debreu's method. However, for any fixed $e$, there exists an $L$ which violates 
the positivity of $e-\operatorname{proj}_{L}(e)$.

The following solution is proposed. To insure monotonicity of the (projectionbased) constructed preference ordering, we have to alter the point $e(L)$, from which the projection is taken, according to $L$, so that $e(L)-\operatorname{proj}_{L}(e(L))$ is always positive. We do this by considering projection from a set $A$, where we imagine $A$ to be a "large" closed ball in $R_{+}^{3}$ (a formal definition is given in the next section). Let $\operatorname{proj}_{L}(A)$ be the point in $L$ closest to $A$ and let $e(L)$ be the point in $A$ closest to $L$. Evidently $\operatorname{proj}_{L}(A)=\operatorname{proj}_{L}(e(L))$. It is not difficult to see that $e(L)-\operatorname{proj}_{L}(A)$ is always positive for $L$ in a compact set when $A$ is sufficiently large. See Figure 1 and compare projections from $e$ and $A$.

The proof in the next section is in essence a reconstruction of the argument of Debreu (1974), based on projection from the set $A$ rather than projection from one given point $e$. Some technical difficulties must be overcome.

\section{Proof of Theorem}

The proof consists of three lemmas. In Lemmas 1 and 2, we decompose a candidate function. In Lemma 3, we show that each decomposed function can be interpreted as a reasonable individual excess demand.

We start with the definition of the set $A \subset R^{n}$, which is central to the proof. The metric on $R^{n}$ is given by the Euclidean norm $\|\cdot\|$, and for $X, Y \subset R^{n}$, the distance between these sets is defined as $d(X, Y)=i n f_{x \in X, y \in Y}\|x-y\|$. We write Int $X$ and $\partial X$ to denote the interior of $X$ and the boundary of $X$, respectively. Let $\Omega$ denote the collection of all sets $A \subset R^{n}$ such that

(A1) $A$ is closed;

(A2) if $x, x^{\prime} \in A, x \neq x^{\prime}$, then $r x+(1-r) x^{\prime} \in \operatorname{Int} A$ for $0<r<1$;

(A3) $x+R_{+}^{n} \subset A$ for $x \in A$;

(A4) $A \subset R_{++}^{n}$; and

(A5) for any $\epsilon>0$ and $x \in \partial R_{+}^{n} \backslash 0$, there exists $r>0$ such that $d(A, r x)<\epsilon$.

An example of $A \in \Omega$ is exhibited in the proof of Lemma 1 .

For given $A \in \Omega$ and $L \in G_{++}^{k}\left(R^{n}\right)$, let $\bar{a} \in A$ and $\bar{b} \in L$ be the points which give the distance between $A$ and $L$. That is, $(\bar{a}, \bar{b}) \in \arg \min _{a \in A, b \in L}\|a-b\|$. This minimization is well defined, because $A$ and $L$ are closed subsets of $R^{n}$, and, moreover, such $\bar{a}$ and $\bar{b}$ are determined uniquely by the strict convexity 
property (A2). We define the functions on $\Omega \times G_{++}^{k}\left(R^{n}\right)$ that select $\bar{a}$ and $\bar{b}$ as $a(A, L)$ and $b(A, L)$ respectively.

Evidently, $a(A, \cdot)$ and $b(A, \cdot)$ are continuous for fixed $A$, and $(a(A, L)-$ $b(A, L)) \in R_{++}^{n} \bigcap L^{\perp}$ by (A2) and (A3). Note that if $b(A, L)=b\left(A, L^{\prime}\right)$, then $a(A, L)=a\left(A, L^{\prime}\right)$ by (A2). Therefore, if, in addition, these $L$ and $L^{\prime}$ are elements of $G$ satisfying $(*)$, then $L=L^{\prime}$ follows from $a(A, L)=a\left(A, L^{\prime}\right)$.

Let $\bar{k}=k+1$. For $A^{i} \in \Omega, i=1, \ldots, \bar{k}$, and $L \in G_{++}^{k}\left(R^{n}\right)$, we define $B\left(A^{1}, \ldots, A^{\bar{k}}, L\right)$ as the interior of the convex hull of $\bigcup_{i=1}^{\bar{k}} b\left(A^{i}, L\right)$ on $L$ :

$$
B\left(A^{1}, \ldots, A^{\bar{k}}, L\right)=\left\{\begin{array}{l|l}
x \in L & \begin{array}{l}
x=\sum_{i=1}^{\bar{k}} r^{i} b\left(A^{i}, L\right) \\
\text { where } r^{i}>0 \text { and } \sum_{i=1}^{\bar{k}} r^{i}=1
\end{array}
\end{array}\right\} .
$$

Lemma 1 claims that there exists a collection of $A \in \Omega$ such that 0 is always included in one of the above defined sets defined for the $A$ 's.

Lemma 1. Let $G$ be a compact subset of $G_{++}^{k}\left(R^{n}\right)$. Then there exists an integer $m$, open connected sets $B_{j} \subset G_{++}^{k}\left(R^{n}\right)$ and $\left(A^{j 1}, \ldots, A^{j \bar{k}}\right) \in \Omega \times \ldots \times \Omega$, $j=1, \ldots, m$, satisfying

(i) $G \subset \bigcup_{j=1}^{m} B_{j}$

(ii) $\quad 0 \in B\left(A^{j 1}, \ldots, A^{j \bar{k}}, L\right)$ for $L \in \overline{B_{j}}$,

where $\overline{B_{j}}$ is the closure of $B_{j}$.

Proof. For given $L \in G$, take $A^{1}, \ldots, A^{\bar{k}} \in \Omega \times \ldots \times \Omega$ so that $0 \in B\left(A^{1}, \ldots, A^{\bar{k}}, L\right)$ and that $B\left(A^{1}, \ldots, A^{\bar{k}}, L\right)$ is relatively open in $L$. The existence of such $A^{1}, \ldots, A^{\bar{k}}$ is shown as follows. Take $y^{i} \in L, i=1, \ldots, \bar{k}$ so that 0 is enclosed by $y^{i}$ 's on $L: 0=\sum_{i=1}^{\bar{k}} r^{i} y^{i}$ where $r^{i}>0$ and $\sum_{i=1}^{\bar{k}} r^{i}=1$. We will find $A^{i}$ such that $b\left(A^{i}, L\right)=y^{i}$. For each $i$, let $\bar{y}^{i} \in R_{++}^{n}$ be a point such that $\bar{y}^{i}-y^{i}$ is positive and perpendicular to $L$, and let $P^{i}$ be the $(n-1)$ dimensional plane including $\bar{y}^{i}$ and perpendicular to $\bar{y}^{i}-y^{i}$. We let $U^{i}: R^{n} \rightarrow R$ be the Cobb-Douglas function such that $\bar{y}^{i}$ is the maximizer of the function when $P^{i}$ is the feasible set, ${ }^{3}$ and define $A^{i}=\left\{x \in R^{n} \mid U^{i}(x) \geq U^{i}\left(\bar{y}^{i}\right)\right\}$. It is evident from the construction that this $A^{i}$ is strictly supported by $P^{i}$ at $\bar{y}^{i}$, and hence $b\left(A^{i}, L\right)=y^{i}$ as desired. Moreover, the $A^{i}$ evidently satisfies (A1)-(A5).

\footnotetext{
${ }^{3}$ The $U^{i}$ is actually given by $U^{i}(x)=\sum_{l=1}^{n} \alpha_{l}^{i} \ln x_{l}$ where $\alpha_{l}^{i}=\frac{\left(\bar{y}_{l}^{i}\right)^{2}}{\sum_{l^{\prime}}\left(\bar{y}_{l^{\prime}}\right)^{2}}$
} 
From the continuity of $b\left(A^{i}, \cdot\right), 0 \in B\left(A^{1}, \ldots, A^{\bar{k}}, L^{\prime}\right)$ is satisfied for $L^{\prime}$ in a neighborhood $B_{L}$ of $L$. Without loss of generality, we can assume that $0 \in B\left(A^{1}, \ldots, A^{\bar{k}}, L^{\prime}\right)$ for $L^{\prime} \in \overline{B_{L}}$ by taking $B_{L}$ to be sufficiently small.

Considering $L$ itself as the index and adapting the above discussion for every $L \in G$, we obtain $\left\{\left(A^{L 1}, \ldots, A^{L \bar{k}}\right), B_{L}\right\}_{L \in G}$, so that $0 \in B\left(A^{L 1}, \ldots, A^{L \bar{k}}, L^{\prime}\right)$ for $L^{\prime} \in \overline{B_{L}}$. Since $\left\{B_{L}\right\}_{L \in G}$ is an open cover of a compact set $G$, we can choose a finite cover, that is, we can choose $L(1), \ldots, L(m)$ so that $G \subset \bigcup_{j=1}^{m} B_{L(j)}$. Rewriting $L(j)$ as $j$, we obtain the statement of the lemma.

Thus we obtain a collection of $A \in \Omega$ numbering $\bar{k} \times m$. This is the number of consumers needed for our decomposition. ${ }^{4}$

Lemma 2. Assume the conditions of Lemma 1. Let $z: G \rightarrow R^{n}$ be a continuous function such that $z(L) \in L$. Then there exist positive valued continuous functions $\beta^{j i}: G \rightarrow R_{++}, j=1, \ldots, m, i=1, \ldots, \bar{k}$, so that, for $L \in G$,

$$
z(L)=\sum_{j, i} \beta^{j i}(L) b\left(A^{j i}, L\right) .
$$

Proof. We exhibit a method for constructing $\beta^{j i}$. Pick any $\epsilon>0$, and define a continuous function $\psi: G_{++}^{n}\left(R^{n}\right) \rightarrow R^{n}$ by $\psi(L)=\epsilon \sum_{j=1}^{m} \sum_{i=1}^{\bar{k}} b\left(A^{j i}, L\right)$.

Recall Lemma 1. Since $B\left(A^{j 1}, \ldots, A^{j \bar{k}}, L\right)$ is relatively open in $L, z(L)-$ $\psi(L)$, which is on $L$, is decomposed uniquely as $z(L)-\psi(L)=\sum_{i} c^{j i}(L) b\left(A^{j i}, L\right)$ with $c^{j i}(L) \in R$ satisfying $\sum_{i} c^{j i}(L)=1$ for $L \in \overline{B_{j}} \bigcap G$. Similarly, 0 is written uniquely as $0=\sum_{i} c_{0}^{j i}(L) b\left(A^{j i}, L\right)$ where $\sum_{i} c_{0}^{j i}(L)=1$ for $L \in \overline{B_{j}} \cap G$. Note that the functions $L \mapsto c^{j i}(L)$ and $L \mapsto c_{0}^{j i}(L)$ are continuous, because $L \mapsto b\left(A^{j i}, L\right)$ and $L \mapsto z(L)-\psi(L)$ are continuous. Note also that every $c_{0}^{j i}(L)$ is positive, because 0 is included in $B\left(A^{j 1}, \ldots, A^{j \bar{k}}, L\right)$ for $L \in \overline{B_{j}}$. Therefore, by defining $\hat{\beta}^{j i}(L)=c^{j i}(L)+t c_{0}^{j i}(L)$ with sufficiently large $t>0$, we obtain continuous, positive valued functions $\hat{\beta}^{j i}: \overline{B_{j}} \bigcap G \rightarrow R_{++}$such that

$$
z(L)-\psi(L)=\sum_{i=1}^{\bar{k}} \hat{\beta}^{j i}(L) b\left(A^{j i}, L\right), \quad \text { for } L \in \overline{B_{j}} \bigcap G
$$

Note that $\hat{\beta}^{j i}$, and hence the right hand side of (1), is defined only on $\overline{B_{j}} \bigcap G$.

\footnotetext{
${ }^{4}$ Though $m$ is determined by $k, n$ and $G$ as shown in Lemma 1, it is not possible to write down the relation in an explicit formula.
} 
We extend $\hat{\beta}^{j i}$ to a continuous function $\tilde{\beta}^{j i}: G \rightarrow R_{++}$with $\tilde{\beta}^{j i} \mid B_{j} \bigcap G=$ $\hat{\beta}^{j i}$. Such an extension is possible because $\hat{\beta}^{j i}$ is a continuous function on a compact set (Royden, 1988 [p.179]). Of course

$$
z(L)-\psi(L)=\sum_{i=1}^{\bar{k}} \tilde{\beta}^{j i}(L) b\left(A^{j i}, L\right), \quad \text { for } L \in \overline{B_{j}} \bigcap G,
$$

and now both sides of the equality of (2) are functions defined on $G$.

Recall that $\left\{B_{j}\right\}_{j=1}^{m}$ is a finite open cover of the compact subset $G$ of the smooth compact manifold $G^{k}\left(R^{n}\right)$. Obviously $\bigcup_{j} B_{j}$ is a submanifold of $G^{k}\left(R^{n}\right)$ and $\left\{B_{j}\right\}_{j=1}^{m}$ is its open cover. Thus there exists a smooth partition of unity $\left\{\varphi_{j}\right\}_{j=1}^{m}$ on $\bigcup_{j} B_{j}$ subordinate to $\left\{B_{j}\right\}_{j=1}^{m}$ (Hirsh, $\left.1976[\mathrm{p} .43]\right)$. In other words, each $\varphi_{j}: \bigcup_{j} B_{j} \rightarrow R$ is smooth; $0 \leq \varphi_{j} \leq 1 ; \operatorname{support}\left(\varphi_{j}\right) \subset B_{j}$; $\sum_{j=1}^{m} \varphi_{j} \equiv 1$. Then, for $L \in G$,

$$
\begin{aligned}
z(L)-\psi & (L)=\sum_{j=1}^{m} \varphi_{j}(L)(z(L)-\psi(L)) \\
= & \sum_{j=1}^{m} \varphi_{j}(L) \sum_{i=1}^{\bar{k}} \tilde{\beta}^{j i}(L) b\left(A^{j i}, L\right) \\
= & \sum_{j, i} \varphi_{j}(L) \tilde{\beta}^{j i}(L) b\left(A^{j i}, L\right),
\end{aligned}
$$

where in the second equality we used the fact that $\sum_{i=1}^{\bar{k}} \tilde{\beta}^{j i}(L) b\left(A^{j i}, L\right) \neq$ $z(L)-\psi(L) \Rightarrow \varphi_{j}(L)=0$. Actually, if the left hand side of this arrow is satisfied, then $L \notin \overline{B_{j}} \cap G$ from (2). Then $L \in G \backslash \overline{B_{j}}$ for $L$ in $G$, and hence $\varphi_{j}(L)=0$.

From the definition of $\psi(L), z(L)=\sum_{j, i}\left(\varphi_{j}(L) \tilde{\beta}^{j i}(L)+\epsilon\right) b\left(A^{j i}, L\right)$. Define $\beta^{j i}(L)=\varphi_{j}(L) \tilde{\beta}^{j i}(L)+\epsilon$. This function is positive valued and continuous.

From the previous lemmas, we can decompose any continuous function $z$ defined on $G$ such that $z(L) \in L$ into $z^{j i}(L)=\beta^{j i}(L) b\left(A^{j i}, L\right), j=1, \ldots, m$, $i=1, \ldots, \bar{k}$. In Lemma 3 we construct the individual preference ordering so that $z^{j i}(L)$ is the excess demand for $L$. It will be clear that this lemma ends the proof of our theorem. Since considering one, say $(j, i)$-th, consumer is sufficient, we omit the index $(j, i)$ and simply write $z(L)=\beta(L) b(A, L)$. This individual candidate excess demand function should not be confused with the aggregate excess demand we have dealt with.

Lemma 3. Let $G$ be a compact subset of $G_{++}^{k}\left(R^{n}\right)$ satisfying $(*)$, and let $z: G \rightarrow R^{n}$ be a function defined by $z(L)=\beta(L) b(A, L)$, where $\beta$ is a positive 
valued continuous function defined on $G$ and $A \in \Omega$. Then there exists a consumer $(\preceq, \omega)$ such that $z(L)+\omega$ is the greatest element for $\preceq$ in $L-R_{+}^{n}$.

Proof. The proof consists of Steps 0-3. Step 0 gives a lemma which generalizes part of a discussion in Debreu (1974). Steps 1 and 2 construct the framework to which the lemma is then applied in Step 3.

Step 0. The discussion of Debreu (1974) [p.18 1.14-p.20 1.16] is summarized in the next lemma. This lemma gives a sufficient condition of the framework so that a preference ordering with the required properties is constructed on it. In the sequel, the set of non-empty, closed subsets of $R^{n}$ is endowed with the Hausdorff topology. We start from the definition of preference-or-indifference sets, which represent a preference ordering. We call sets $Q_{t} \subset R_{+}^{n}$ defined for $t \geq 0$ preference-or-indifference sets whenever they satisfy (P1) $Q_{t}$ is closed; $t \mapsto Q_{t}$ is continuous, (P2) $x+R_{+}^{n} \subset Q_{t}$ for $x \in Q_{t} ; Q_{t^{\prime}} \cap R_{++}^{n} \subset \operatorname{Int} Q_{t}$ for $t \leq t^{\prime}$, (P3) if $p, q \in Q_{t} \cap R_{++}^{n}$ and $p \neq q, \lambda p+(1-\lambda) q \in$ Int $Q_{t}$ for $\lambda \in(0,1)$, (P4) for any $x \in R_{+}^{n}$ there exists $t^{\prime}$ such that $x \in \partial Q_{t^{\prime}}$. It is clear that $(\mathrm{P} 1)-(\mathrm{P} 4)$ imply respectively continuity, monotonicity, strict convexity and completeness of the preference ordering represented by $Q_{t}, t \geq 0$.

Lemma A. Suppose a segment $\left[t_{1}, t_{2}\right] \subset R_{+}$and an $n-1$ dimensional plane $H$ strictly supporting $R_{+}^{n}$ at 0 are given. Let $K_{t} \subset R^{n}$ and $C_{t} \subset R^{n}$ defined for $t \in\left[t_{1}, t_{2}\right]$ satisfy the following properties.

(i) $K_{t}$ is closed and convex; $t \mapsto K_{t}$ is continuous; $0 \in K_{t} ; K_{t^{\prime}} \backslash 0 \subset \operatorname{Int} K_{t}$, for $t<t^{\prime}$, (ii) $x+R_{+}^{n} \subset K_{t}$ for $x \in K_{t}$, (iii) if $p, q \in \partial K_{t}, p \neq q$ and $p-q \in H$, then $\lambda p+(1-\lambda) q \in$ Int $_{t}$ for $\lambda \in(0,1)$, (iv) $H$ strictly supports $K_{t}$ at 0 , (v) $C_{t} \subset \partial K_{t}$, (vi) $C_{t}$ is compact; $t \mapsto C_{t}$ is continuous; $0 \notin C_{t}$ for $t \in\left(t_{1}, t_{2}\right]$, and (vii) if $x, x^{\prime} \in C_{t}$ and $x \neq x^{\prime}$, then $\lambda x+(1-\lambda) x^{\prime} \in \operatorname{Int}_{t}$ for $\lambda \in(0,1)$.

Then there exist preference-or-indifference sets $Q_{t} \subset R_{+}^{n}, t \geq 0$,and $\omega \in R_{+}^{n}$ satisfying (I) $\left(Q_{t}-\omega\right) \bigcap \partial K_{t}=C_{t}$ and (II) $\left(Q_{t}-\omega\right) \subset K_{t}$, for $t \in\left[t_{1}, t_{2}\right]$.

Proof. Apply the discussion of Debreu (1974) [p.18 1.14-p.20 1.16]: replace $\alpha, \omega, L_{t}^{*}, e^{i}$ and $l$ there by $t_{1}, t_{2}, K_{t}, \omega$ and $n$ and construct $Q_{t}$ and $\omega$, starting from $D_{t}=\bigcup_{t<s \leq t_{2}} C_{s}$ and following the discussion. The discussion holds word for word. (Trivial exceptions are that we do not need the construction of $H$ [p.19 1.9-11 Debreu (1974)] and that the consequences of the arguments [p.20 1.1-4 Debreu (1974)] and [p.20 1.5-8 Debreu (1974)] directly follow from 
the assumptions (vii) and (iii) respectively.) The statements (I) and (II) are evident from the construction.

Note that $K_{t}$ in Lemma $\mathrm{A}$ is not assumed to be a strictly convex cone, whereas the corresponding set $L_{t}^{*}$ in Debreu (1974) is. Instead we assume (iii) and (vii), which are implied by Debrue's strict convex cone assumption.

Step 1: This step gives the basic framework by which the preference-orindifference set $Q_{t}$ is constructed. Let $\bar{x}$ be the point in $A$ closest to 0 . That is, $d(A, 0)=d(\bar{x}, 0)=\|\bar{x}\|$. Define $H$ and $H_{+}$by $H=\bar{x}^{\perp}=\left\{x \in R^{n} \mid x \cdot \bar{x}=0\right\}$ and $H_{+}=\left\{x \in R^{n} \mid x \cdot \bar{x} \geq 0\right\}$ respectively.

Let $\alpha=\min _{L \in G} d(A, L)$. Since every $L$ includes $0, d(A, L) \leq d(A, 0)=\|\bar{x}\|$ for any $L \in G$. Therefore, $\alpha \leq d(A, L) \leq\|\bar{x}\|$, and equivalently $\frac{1}{\|\bar{x}\|} \leq \frac{1}{d(A, L)} \leq$ $\frac{1}{\alpha}$ for any $L \in G$. We define $t_{1}=\frac{1}{\|\bar{x}\|}$ and $t_{2}=\frac{1}{\alpha}$.

For every $t \in\left[t_{1}, t_{2}\right]$, define $V_{t} \subset G$ as the set of $L \in G$ at distance $\frac{1}{t}$ from A. That is,

$$
V_{t}=\left\{L \in G \mid \frac{1}{d(A, L)}=t\right\} .
$$

Since the consumer determines her demand when $L$ is given, we can consider the indirect utility to be a function of $L$. We will construct a preference ordering so that $V_{t}$ is the indirect indifference class in $G$.

For $x \in R^{n} \backslash 0$, we write the ray which ends at 0 and passes through $x$ as $[x]$. That is, $[x]=\left\{y \in R^{n} \mid y=r x, r \geq 0\right\}$. Define $H_{t}$ for $t \in\left(t_{1}, t_{2}\right]$ as the subset of $R^{n}$ defined by the union of rays at distance less than or equal to $\frac{1}{t}$ from $A$ and define $H_{t_{1}}$ as the limit of $H_{t}$ as $t \rightarrow t_{1}$. Formally,

$$
H_{t}=\left\{\begin{array}{l}
\bigcup_{d(y, A) \leq \frac{1}{t}} y: y \text { is a ray whose end point is } 0 \text { for } t \in\left(t_{1}, t_{2}\right] . \\
H_{+} \text {for } t=t_{1}
\end{array} .\right.
$$

Note that, for $t \in\left(t_{1}, t_{2}\right], H_{t}$ is a strictly convex cone by the strict convexity property (A2) of $A$, and that $\partial H_{t}$ is the subset of $R^{n}$ defined by the union of rays at distance exactly $\frac{1}{t}$ from $A$. It is clear that $H_{t}$ is convex and closed, $t \mapsto H_{t}$ is continuous, and $H_{t^{\prime}} \backslash 0 \subset \operatorname{Int} H_{t}$ for $t<t^{\prime}$. Also, $R_{+}^{n} \backslash 0 \subset$ Int $H_{t}$ by (A4).

Since $A$ is fixed, we write $b(A, L)$ as $b(L)$ for simplicity. Observe that $z(L)=\beta(L) b(L) \in \partial H_{t}$ for each $t \in\left[t_{1}, t_{2}\right]$ and $L \in V_{t}{ }^{5}$ Actually, it is clear

\footnotetext{
${ }^{5}$ Rigorously speaking, the trivial condition "if $V_{t}$ is nonempty" should be given. However, to avoid unnecessary complication of the statement, we do not mention this condition hereafter.
} 
from the definition of $V_{t}$ and $H_{t}$ that, for each $t \in\left(t_{1}, t_{2}\right], L \in V_{t}$ comes in contact with $H_{t}$ along the ray $[b(L)]$ (see Figure 2), and hence $z(L) \in \partial H_{t}$. For $t=t_{1}, b(L)=0$ for $L \in V_{t_{1}}$, and hence $z(L)=0 \in \partial H_{t_{1}}=H$. Therefore, $z\left(V_{t}\right) \subset \partial H_{t}$ for every $t \in\left[t_{1}, t_{2}\right]$.

Step 2: We cannot apply Lemma A directly to $H_{t}$ and $z\left(V_{t}\right)$ because they do not satisfy (i)-(vii). First, $t \mapsto z\left(V_{t}\right)$ is not necessarily continuous because $t \mapsto V_{t}$ is not necessarily continuous with respect to the Hausdorff topology on the set of closed subsets of (suitably metrized) $G$. Second, $H_{t_{1}}$ obviously does not satisfy (iii) and (iv). The purpose of this step is to construct $C_{t}$ and $K_{t}$ such that (i)-(vii) in Lemma A hold, and, in addition, both $z\left(V_{t}\right) \subset C_{t}$ and $K_{t} \subset H_{t}$ hold. We exhibit a method to construct such $C_{t}$ and $K_{t}$. The proof that they satisfy (i)-(vii) is given in Appendix. It is almost evident from the construction.

Construction of $C_{t}$. We extend $z\left(V_{t}\right)$ to $C_{t}$ as follows. Let

$$
\begin{aligned}
& T_{t}=\left\{b(L) \in R^{n} \mid L \in V_{t}\right\}, \\
& \tilde{T}_{t}=\left\{\operatorname{proj}_{y}(A) \in R^{n} \mid y \text { is a ray whose end point is } 0 \text { and } y \subset \partial H_{t}\right\},
\end{aligned}
$$

where $\operatorname{proj}_{y}(A)$ denotes the point on $y$ closest to $A$, and let $T=\bigcup_{t_{1} \leq t \leq t_{2}} T_{t}$, $\tilde{T}=\bigcup_{t_{1} \leq t \leq t_{2}} \tilde{T}$. Recall the definition of $V_{t}$ and $H_{t}$. Evidently $T_{t}, \tilde{T}_{t}, T, \tilde{T}$ are compact subsets of $R^{n} ; T_{t} \subset \tilde{T}_{t} ; T \subset \tilde{T}$.

We claim that $L \mapsto b(L)$ is a homeomorphism from $G$ to $T$. It is sufficient to show that $L \mapsto b(L)$, which is evidently continuous and onto, is also one-toone, because $G$ and $T$ are compact sets in Hausdorff spaces (Royden, 1988 [pp. 191-192]). But this (i.e., $b(L)=b\left(L^{\prime}\right) \Rightarrow L=L^{\prime}$ ) has already been established before Lemma 1.

Now we write the inverse function of $L \mapsto b(L)$ as $b^{-1}: T \rightarrow G$, define $\bar{\beta}: T \rightarrow R_{+}$as $\bar{\beta}=\beta \circ b^{-1}$, and let $\tilde{\beta}: \tilde{T} \rightarrow R_{+}$be a continuous extension of $\bar{\beta}$, that is, $\tilde{\beta} \mid T=\bar{\beta}$. Such an extension exists because $\bar{\beta}$ is a continuous function on a compact set. Let $\tilde{z}: \tilde{T} \rightarrow R^{n}$ be defined by $\tilde{z}(x)=\tilde{\beta}(x) x$, and let $C_{t}=\tilde{z}\left(\tilde{T}_{t}\right)$ for $t \in\left[t_{1}, t_{2}\right]$. This is the definition of $C_{t}$. We also define $D_{t}=\bigcup_{t \leq s \leq t_{2}} C_{s}$.

That $z\left(V_{t}\right) \subset C_{t}$ follows from the fact that $z(L)=\beta(L) b(L)=\tilde{\beta}(b(L)) b(L)=$ $\tilde{z}(b(L))$, and that $b(L) \in T_{t} \subset \tilde{T}_{t}$ when $L \in V_{t}$. The following properties are also clear. First, $C_{t}=\tilde{z}\left(\tilde{T}_{t}\right) \subset \partial H_{t}$ from the definition of $\tilde{T}_{t}$. Second, 
$C_{t_{1}}=\tilde{z}\left(\tilde{T}_{t_{1}}\right)=0$, because $\operatorname{proj}_{y}(A)=0$ for a ray such that $y \subset \partial H_{t_{1}}$. Third, $D_{t_{1}}=\bigcup_{t_{1} \leq t \leq t_{2}} C_{t}=\tilde{z}(\tilde{T})$ is compact and strictly supported by $H$ at 0 .

Construction of $K_{t}$. We construct $K_{t}$ by slightly modifying $H_{t}$. Let $K$ be a closed convex set satisfying (ii)-(iv), with $K_{t}$ replaced by $K$ and $D_{t_{1}} \backslash 0 \subset$ Int $K$. For example, define $K$ as $K=\tilde{H}+B$ where $\tilde{H}$ is a strictly convex cone including $R_{+}^{n}$ and strictly supported by $H$ at 0 , and $B$ is a closed ball tangent to $H$ at 0 and including $D_{t_{1}} \backslash 0$ in its interior.

For every $q \in H$, let $\lambda_{t}(q)$ be the least $r$ such that $q+r \bar{x} \in H_{t}, \gamma(q)$ be the least $r$ such that $q+r \bar{x} \in K$, and let $\mu_{t}(q)$ be defined by

$$
\mu_{t}(q)=\left\{\begin{array}{ll}
\lambda_{t}(q) & \text { if } \lambda_{t}(q) \geq \gamma(q) \\
\frac{1}{2}\left(\lambda_{t}(q)+\gamma(q)\right) & \text { if } \lambda_{t}(q) \leq \gamma(q)
\end{array} .\right.
$$

We define $K_{t}$ as the set of $q+r \bar{x}$ where $q \in H$ and $r \geq \mu_{t}(q)$. That $K_{t} \subset H_{t}$ is clear.

Step 3. By applying Lemma A to the $K_{t}$ and $C_{t}$ constructed in Step 2, we obtain the direct preference-or-indifference sets $Q_{t}, t \geq 0$, and the endowment $\omega \in R_{+}^{n}$ satisfying (I) and (II). We see that these possess the desired property: $z(L)$ is the demand for $L$. Consider the intersection of $Q_{t}-\omega, L$ and $K_{t}$. It is clear from (I), $z\left(V_{t}\right) \subset C_{t}$ and $z(L) \in L$ that $z(L) \in\left(Q_{t}-\omega\right) \bigcap L \bigcap K_{t}$ for $L \in V_{t}$. On the other hand, recall that $L \in V_{t}$ contacts $H_{t}$ on a ray as drawn in Figure 2. Since $\left(Q_{t}-\omega\right) \subset K_{t} \subset H_{t}$ from $K_{t} \subset H_{t}$ and (II), the surface $\partial H_{t}$ (and $\partial K_{t}$ ) separates $Q_{t}-\omega$ and $L \in V_{t}$. From the linearity of $L$ and strict convexity (P3) of $Q_{t}$, this means that $z(L)$ is the unique intersection of $L \in V_{t}$ and $Q_{t}-\omega$. This ends the proof of Lemma 3 .

\section{A Appendix}

We prove that the $C_{t}$ and $K_{t}$ in Step 2 satisfy (i)-(vii) in Lemma A.

Proof of (vi): From the definition of $\tilde{T}_{t}$, it is clear that $t \mapsto \tilde{T}_{t}$ is continuous. It is also clear that $\tilde{z}: \tilde{T} \rightarrow R^{n}$ is continuous. The continuity of $t \mapsto C_{t}=\tilde{z}\left(\tilde{T}_{t}\right)$ follows. The other properties in (vi) are clear.

Proof of (i), (ii) and (iv): Let $q \in H$. If $\lambda_{t}(q) \leq \gamma(q)$, then $\mu_{t}(q)=$ $\frac{1}{2}\left(\lambda_{t}(q)+\gamma(q)\right) \geq \lambda_{t}(q)$. If $\lambda_{t}(q) \leq \gamma(q)$, then $\mu_{t}(q)=\frac{1}{2}\left(\lambda_{t}(q)+\gamma(q)\right) \geq \lambda_{t}(q)$. Therefore, $\mu_{t}(q) \geq \max \left\{\lambda_{t}(q), \frac{1}{2}\left(\lambda_{t}(q)+\gamma(q)\right)\right\}$, whereas $\mu_{t}(q)$ is either $\lambda_{t}(q)$ 
or $\frac{1}{2}\left(\lambda_{t}(q)+\gamma(q)\right)$ from the definition. Thus

$$
\mu_{t}(q)=\max \left\{\lambda_{t}(q), \frac{1}{2}\left(\lambda_{t}(q)+\gamma(q)\right)\right\} .
$$

The convexity of $\mu_{t}$ now follows from that of $\lambda_{t}$ and $\frac{1}{2}\left(\lambda_{t}+\gamma\right)$. This establishes the convexity of $K_{t}$. The other properties in (i), (ii) and (iv) are immediate.

Proof of (v): Recall that $C_{t}$ is on $\partial H_{t}$. Therefore, for each $x^{\prime} \in C_{t}$, there exists a $q^{\prime} \in H$ such that $x^{\prime}=q^{\prime}+\lambda_{t}\left(q^{\prime}\right) \bar{x}$. Since $K$ includes $C_{t}, \lambda_{t}\left(q^{\prime}\right) \geq \gamma\left(q^{\prime}\right)$. Therefore $\mu_{t}\left(q^{\prime}\right)=\lambda_{t}\left(q^{\prime}\right)$. This implies that $x^{\prime}$ is on $\partial K_{t}$. Therefore $C_{t} \subset \partial K_{t}$.

Proof of (vii): Since $C_{t_{1}}=0$, (vii) is true for $t=t_{1}$. Let $t \in\left(t_{1}, t_{2}\right]$ and pick any $x^{1}, x^{2} \in C_{t}, x^{1} \neq x^{2}$. Then there exist $q^{i} \in H$ such that $x^{i}=q^{i}+\lambda_{t}\left(q^{i}\right) \bar{x}$, $i=1,2$. Note that $\lambda_{t}\left(q^{i}\right) \geq \gamma\left(q^{i}\right), i=1,2$, and that $\left[x^{1}\right] \neq\left[x^{2}\right]$, because $\left[x^{1}\right]=\left[x^{2}\right]$ means $\operatorname{proj}_{\left[x^{1}\right]}(A)=\operatorname{proj}_{\left[x^{2}\right]}(A)$, and hence $x^{1}=\tilde{z}\left(\operatorname{proj}_{\left[x^{1}\right]}(A)\right)=$ $x^{2}$. We want to show that $s x^{1}+(1-s) x^{2} \in \operatorname{Int} K_{t}$ for $s \in(0,1)$, that is, $\mu_{t}\left(s q^{1}+(1-s) q^{2}\right)<s \lambda_{t}\left(q^{1}\right)+(1-s) \lambda_{t}\left(q^{2}\right)$ for $s \in(0,1)$. Write $s q^{1}+(1-s) q^{2}=$ $\bar{q}$.

If $\lambda_{t}(\bar{q}) \geq \gamma(\bar{q})$, then $\mu_{t}(\bar{q})=\lambda_{t}(\bar{q})$. Since $x^{1}, x^{2} \in \partial H_{t}, H_{t}$ is a strictly convex cone, and since $\left[x^{1}\right] \neq\left[x^{2}\right]$, we have $\lambda_{t}(\bar{q})<s \lambda_{t}\left(q^{1}\right)+(1-s) \lambda_{t}\left(q^{2}\right)$.

If $\lambda_{t}(\bar{q})<\gamma(\bar{q})$, then $\mu_{t}(\bar{q})=\frac{1}{2}\left(\lambda_{t}(\bar{q})+\gamma(\bar{q})\right)<\gamma(\bar{q})$. Because of the convexity of $\gamma$ and $\lambda_{t}\left(q^{i}\right) \geq \gamma\left(q^{i}\right), i=1,2$, we get $\gamma(\bar{q}) \leq s \gamma\left(q^{1}\right)+(1-s) \gamma\left(q^{2}\right) \leq$ $s \lambda_{t}\left(q^{1}\right)+(1-s) \lambda_{t}\left(q^{2}\right)$. This ends the proof of (vii).

Proof of (iii): The case $t=t_{1}$ is immediate. Let $t \in\left(t_{1}, t_{2}\right]$, and let $x^{1}, x^{2} \in \partial K_{t}, x^{1} \neq x^{2}$ and $s \in(0,1)$. We have to show that $s x^{1}+(1-s) x^{2} \in$ $\partial K_{t} \Rightarrow x^{1}-x^{2} \in H$. That is, we have to show that $\mu_{t}(\bar{q})=s \mu_{t}\left(q^{1}\right)+(1-$ s) $\mu_{t}\left(q^{2}\right) \Rightarrow \mu_{t}\left(q^{1}\right) \neq \mu_{t}\left(q^{2}\right)$, where $q^{i} \in H$ is defined by $x^{i}=q^{i}+\mu_{t}\left(q^{i}\right) \bar{x}$ for $i=1,2$ and $\bar{q}=s q^{1}+(1-s) q^{2}$. Observe that

$$
\begin{aligned}
& \mu_{t}(\bar{q})=\max \left\{\lambda_{t}(\bar{q}), \frac{1}{2}\left(\lambda_{t}(\bar{q})+\gamma(\bar{q})\right)\right\} \\
& \geq \max \left\{s \lambda_{t}\left(q^{1}\right)+(1-s) \lambda_{t}\left(q^{2}\right), \frac{1}{2}\left(s \lambda\left(q^{1}\right)+(1-s) \lambda_{t}\left(q^{2}\right)+s \gamma\left(q^{1}\right)+(1-s) \gamma\left(q^{2}\right)\right\}\right. \\
& \geq \max \left\{s \lambda_{t}\left(q^{1}\right), s \frac{1}{2}\left(\lambda_{t}\left(q^{1}\right)+\gamma\left(q^{1}\right)\right)\right\}+\max \left\{(1-s) \lambda_{t}\left(q^{2}\right),(1-s) \frac{1}{2}\left(\lambda_{t}\left(q^{2}\right)+\gamma\left(q^{2}\right)\right)\right\} \\
& =s \mu_{t}\left(q^{1}\right)+(1-s) \mu_{t}\left(q^{2}\right),
\end{aligned}
$$

where the second line follows from the convexity of $\lambda_{t}$ and $\gamma$, and the third line follows from positivity of the elements $s \lambda_{t}\left(q^{1}\right),(1-s) \lambda_{t}\left(q^{2}\right)$, etc. From the second line, $\mu_{t}(\bar{q})=s \mu_{t}\left(q^{1}\right)+(1-s) \mu_{t}\left(q^{2}\right)$ only if $\lambda_{t}(\bar{q})=s \lambda_{t}\left(q^{1}\right)+(1-s) \lambda_{t}\left(q^{2}\right)$. This is so if and only if $q^{1}$ and $q^{2}$ is on the same ray, because $H_{t}$ is a strictly 
convex cone. Assume $\left[q^{1}\right]=\left[q^{2}\right]$, and, without loss of generality, let $\left\|q^{1}\right\|<\|$ $q^{2} \|$. Then $\lambda\left(q^{1}\right)<\lambda\left(q^{2}\right)$ and $\gamma\left(q^{1}\right)<\gamma\left(q^{2}\right)$ from the definition of $H_{t}$ and $K$, and hence $\mu_{t}\left(q^{1}\right)<\mu_{t}\left(q^{2}\right)$. This ends the proof of (iii).

\section{References}

[1] J. M. Bottazzi and T. Hens, Excess demand functions and incomplete market, Journal of Economic Theory 68 (1996), 49-63.

[2] P. A. Chappori and I. Ekeland, Disaggregation of excess demand function in incomplete markets, Journal of Mathematical Economics 31 (1999), 111-129.

[3] G. Debreu, Excess demand functions, Journal of Mathematical Economics 1 (1974), 15-23.

[4] P. Gottardi and T. Hens, Disagregation of excess demand and comparative statics with incomplete markets and nominal assets, Econimics Theory 13 (1999), 287-308.

[5] P. Gottardi and A. Mas-Colell, A note on the decomposition (at a point) of aggregate excess demand on the Grassmanian, Journal of Mathematical Economics 33 (2000), 463-473.

[6] T. Hens, An extension of Mantel (1976) to incomplete markets, Journal of Mathematical Economics 36 (2001), 141-149.

[7] M. W. Hirsh, Differential Topology, Springer-Verlarg, New York, 1976.

[8] R. Mantel, On the characterization of aggregate excess demand, Journal of Economic Theory 7 (1974), 348-353.

[9] A. Mas-Colell, Four lectures on the differentiable approach to general equilibrium, in "Mathematical Economics" Lecture Note in Mathematics 1330, 19-49, Springer-Verlag, Berlin, 1986.

[10] H. L. Royden, Real Analysis, third edition, Macmillan, New York, 1988.

[11] H. Sonnenschein, Do Walras' identity and continuity characterize the class of community excess demand functions ? Journal of Economic Theory 6 (1973), 345-354. 


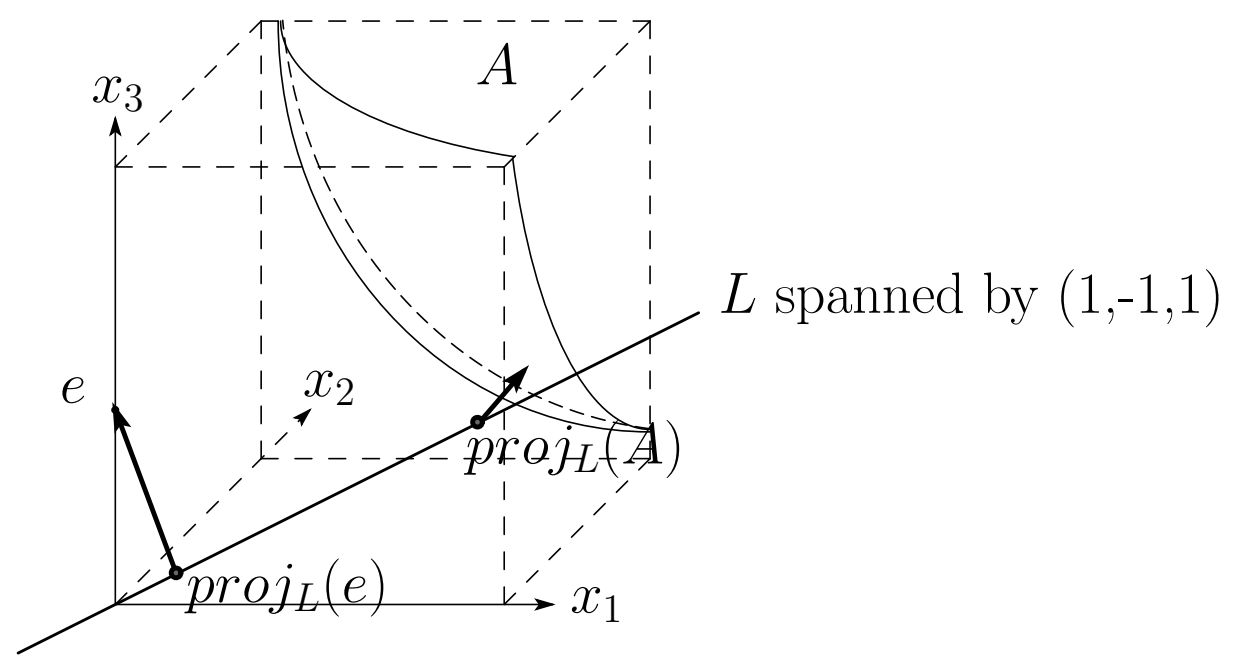

Figure 1: Projection from A 


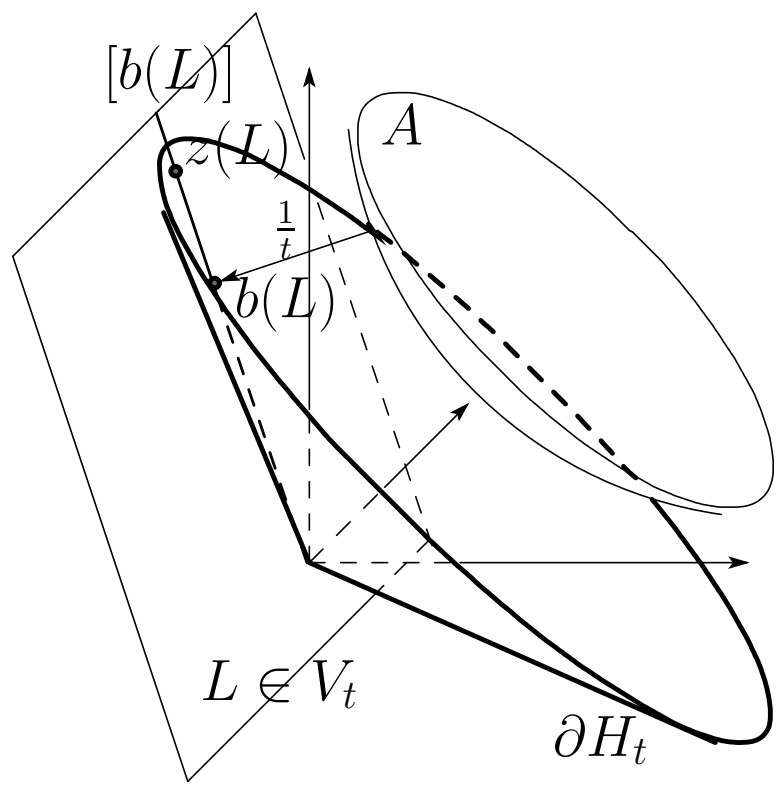

Figure 2: Intersection of $H_{t}$ and $L \in V_{t}$ 